Canadian University Music Review

Revue de musique des universités canadiennes

\title{
Glossolalie : œuvre ouverte
}

\section{François Vanasse}

Numéro 4, 1983

URI : https://id.erudit.org/iderudit/1013899ar

DOI : https://doi.org/10.7202/1013899ar

Aller au sommaire du numéro

\section{Éditeur(s)}

Canadian University Music Society / Société de musique des universités canadiennes

\section{ISSN}

0710-0353 (imprimé)

2291-2436 (numérique)

Découvrir la revue

Citer cet article

Vanasse, F. (1983). Glossolalie : œuvre ouverte. Canadian University Music Review / Revue de musique des universités canadiennes, (4), 95-124.

https://doi.org/10.7202/1013899ar

(c) Canadian University Music Society / Société de musique des universités canadiennes, 1983
Ce document est protégé par la loi sur le droit d'auteur. L’utilisation des services d'Érudit (y compris la reproduction) est assujettie à sa politique d'utilisation que vous pouvez consulter en ligne.

https://apropos.erudit.org/fr/usagers/politique-dutilisation/ 


\title{
GLOSSOLALIE : OEUVRE OUVERTE
}

\author{
François Vanasse
}

Jusqu'au début des années soixante, l'analyse musicale s'est toujours vue confrontée à un corpus bien défini de formes ou de structures fixes, fixité rigoureuse sur le plan graphique (partition), plus fluctuante sur le plan sonore.

C'était là un fait si bien établi que les musicologues en ont fait une condition sine qua non de toute analyse musicale:

La notation musicale, pour le sémiologue, joue deux rôles; ou bien elle est la trace qui rend l'identité de l'oeuvre possible, et dans ce cas, on peut admettre du point de vue de l'analyse qu'elle correspond à son équivalent sonore (c'est la notation prescriptive au sens de Seeger [1958]), ou bien elle est absente ou ne garantit pas le résultat sonore, et il faut bien procéder à une transcription des sons, toujours indispensable, car il n'y a d'analyse "qu'inscrite " (Granger): il lui faut bien un substitut symbolique pour savoir de quoi elle parle (c'est la notation descriptive) (Nattiez 1975:111).

Une des manifestations musicales, devenue courante depuis une vingtaine d'années, nous oblige cependant à remettre en question ce présupposé: il s'agit de l'oeuvre ouverte.

Cet article se propose donc de voir quelques-unes des possibilités qu'offre à l'analyse ce genre d'oeuvre dont les structures varient d'une interprétation à l'autre.

L'analyse n'étant, elle aussi, qu'interprétation de l'oeuvre, nous avons voulu, nous laissant en cela inspirer par l'objet même de notre étude, aborder une seule pièce, la composition Glossolalie de Dieter Schnebel, de plusieurs perspectives différentes. Nous en proposons donc trois modèles qui tentent de rendre compte d'autant d'aspects d'une seule et même partition. 


\section{L'oeuvre}

"Glossolalie » est un mot d'origine grecque qui signifie «don des langues». Le titre fait donc allusion à une variété de langages, variété que l'on retrouvera dans la pièce aussi bien au niveau proprement langagier, où le compositeur demande l'utilisation de plusieurs langues (chinois, allemand, tchèque, etc....), qu'au niveau musical qui est traité comme langage. Le langage, par contre, peut être traité musicalement, ou mieux encore, conçu comme musique.

Toute la pièce est donc construite autour de cette problématique et il serait difficile d'affirmer lequel du langage ou de la musique est prépondérant, Glossolalie se situant plutôt à michemin entre ces deux pôles. La partition que nous étudions est "verbale », c'est-à-dire qu'elle est faite d'un texte, dans lequel le compositeur explique comment il veut que les interprètes construisent sa pièce. ${ }^{1}$ La partition verbale, en effet, ne contient que des directives permettant aux interprètes d'élaborer une autre partition qui, elle, servira à l'exécution sonore de l'oeuvre. Comme le dit le compositeur dans les explications qui précèdent la partition verbale, celle-ci contient des " matériaux préparés . $^{2}$

La partition verbale est constituée de vingt-neuf feuilles séparées, chacune d'elle représentant ce que le compositeur a nommé un tableau. Chaque tableau porte un titre différent, et le matériel que les interprètes auront à utiliser y est défini par des descriptions verbales et graphiques.

Pour construire la partition d'exécution, on peut utiliser tous les tableaux ou seulement quelques-uns de ceux-ci. De plus, l'ordre de succession de ces tableaux est laissé au choix des interprètes. Le compositeur, dans l'explication qui précède la partition, précise cependant qu'on doit éviter une réalisation qui, parce que trop limitée, donnerait une idée restreinte de l'ensemble.

La partition verbale ne détermine donc aucunement la forme finale (sonore) de l'oeuvre qui peut être continue ou discontinue, cyclique ou à déroulement continu, homogène ou hétérogène. De même, la durée du résultat final est indéterminée et peut varier à l'infini.

Chaque tableau se compose de cinq grandes sections délimitant chacune un domaine des matériaux à utiliser. Il y a donc:

- la section d'index matériel (MI),

- la section des caractéristiques matérielles (MC), 
- la section des directives d'actions (AD),

- la section des règles de synchronisation (SR),

- la section de la disposition spatiale (RD).

La première section est graphique et est divisée en déterminations de hauteurs, de durées et de dynamiques.

Les deux sections suivantes (MC et $A D$ ) sont toutes deux divisées en sous-sections vocales (v) et instrumentales (i). (Le lecteur est prié de se reporter à l'exemple 1 pour avoir une idée plus précise de la partition). ${ }^{3}$

L'interprétation des sections graphiques n'est pas rigoureusement codifiée, ce qui donne lieu à d'amples variations dans l'application pratique de celles-ci.

Il nous faut également préciser que toutes les sections ne sont pas nécessairement présentes dans tous les tableaux. Il peut par exemple n'y avoir des précisions que pour la section MC dans un tableau donné, toutes les autres sections étant absentes de ce tableau.

\section{A propos de l'analyse de "Glossolalie »}

On voit donc que Glossolalie est une oeuvre qui ne peut se laisser saisir par l'analyse dans le sens traditionnel du terme. Les problèmes que celle-ci rencontrerait dans la partition que nous étudions sont les suivants:

1-inexistence de la dualité signe-manifestation sonore;

2-modifications des rapports entre les différents paramètres d'organisation d'une exécution à une autre ;

3-multiciplité des codes.

1-Contrairement à la pratique admise en études littéraires où on accepte une variation assez considérable entre le signe (le mot) et ce qu'il représente, le musicologue s'en est toujours tenu à l'analyse offrant une correspondance assez stricte entre le signe musical et sa manifestation sonore. Cette correspondance cependant n'est pas totale, puisque ce qui fait la différence d'une exécution à une autre, c'est justement la variation dans l'interprétation des signes musicaux. Un aspect bien particulier de l'équivalence entre partition et exécution musicale se présente à l'ethnomusicologue, puisqu'un seul corpus peut être noté de bien des manières différentes. ${ }^{4}$

Dans Glossolalie, cependant, cette correspondance n'existe pas. L'auditeur ne peut déterminer quel tableau de la partition verbale et, à plus forte raison, quelle partie de ce tableau les musiciens sont en train de jouer. 


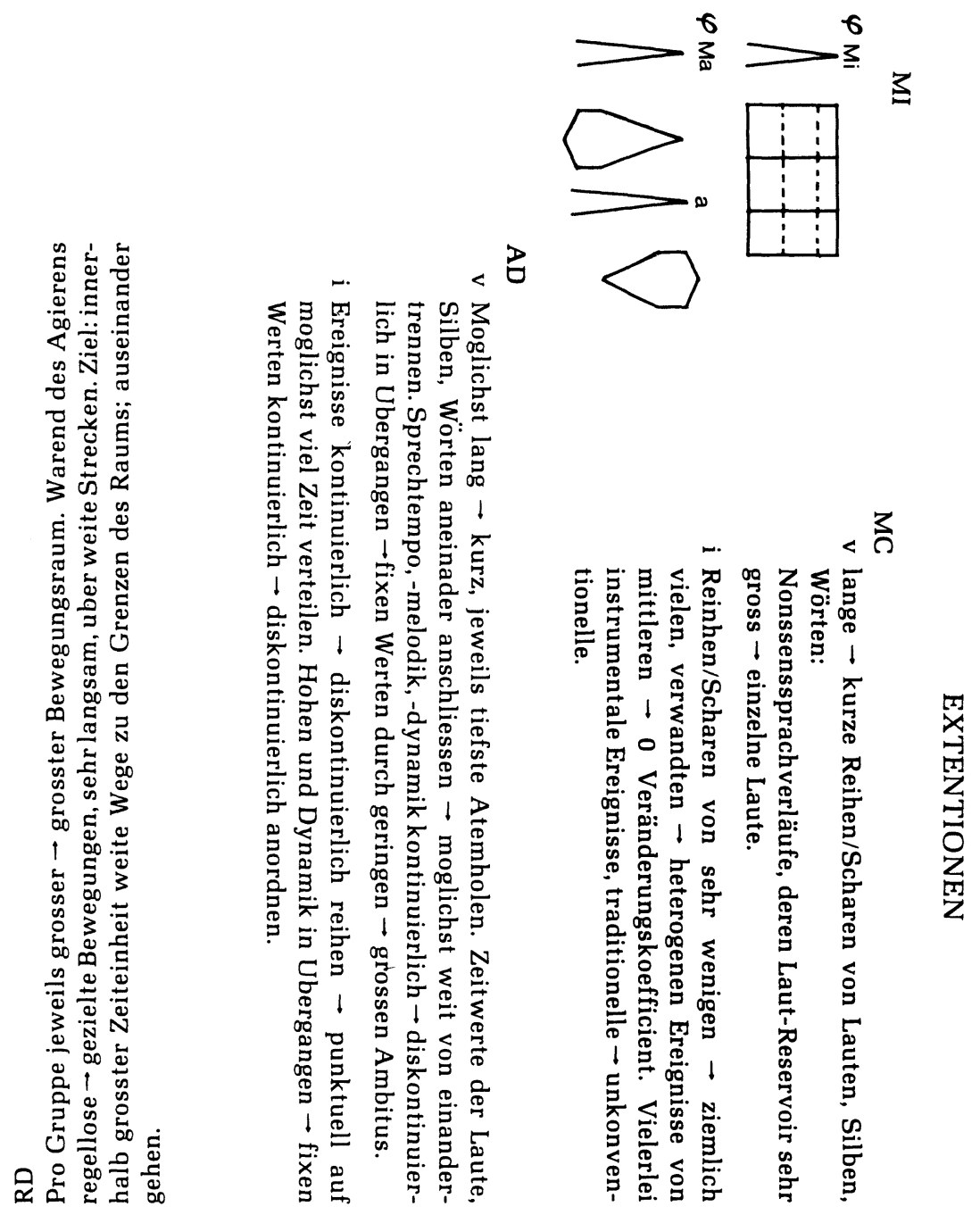

SR

Durch Pausen des Einatmens $\rightarrow$ grossen von anderen abgesetzte Gruppen bilden. Pro Gruppe geringe $\rightarrow$ ziemlich hohe Anzahl von Verlaufen; homogene $\rightarrow$ inhomogene Auswahl, die Verlaufe uberschichten $\rightarrow$ weit auseinandergezogen disponie-renzuzu Zeitraume von geringer $\rightarrow$ geringster Dichte Summen von langsamen $\rightarrow$ langsamsten; einheitliche $\rightarrow$ diffuse Gesamtgestalten.

Einsatze der Verlaufe zugleich $\rightarrow$ nacheinander. Gemeinsam Zeit ausdehnen $\rightarrow$ jeweils langsamst tiefst einatmen, dann moglichst weniger Ereignisse auf moglichst Zeit ver-teilen, dies in moglichst grossen Zeitabstanden von einander. 

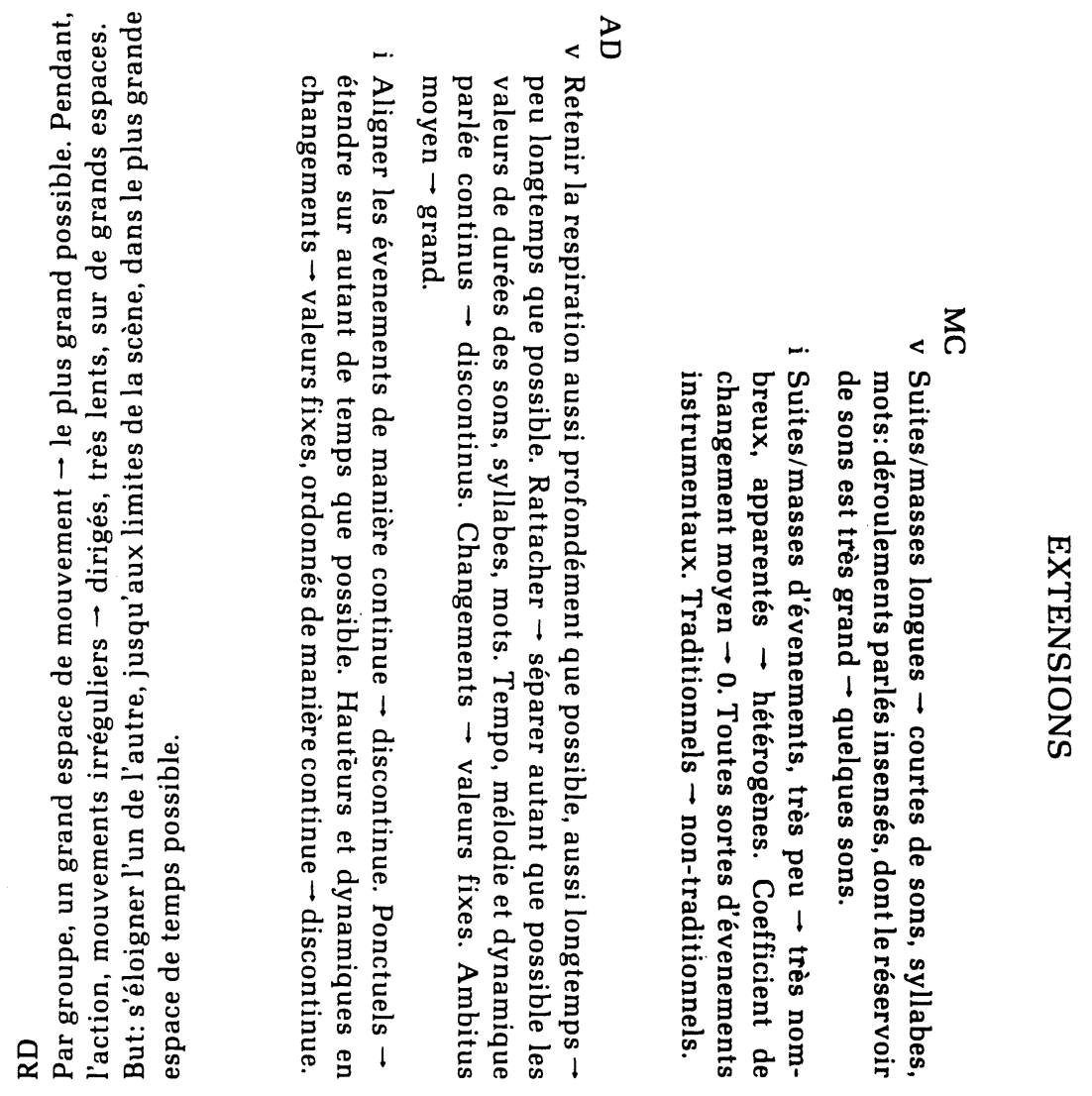

SR

Former des groupes séparés des autres par des pauses de la respiration. Nombre moyen $\rightarrow$ assez grand de déroulements par groupe. Choix homogène $\rightarrow$ hétérogène. Accumuler les évenements en couches $\rightarrow$ les disposer loin l'un de l'autre. Périodes de densité petite $\rightarrow$ très petite. Sommes de formes d'ensembles (Gesamtgestalten) lentes $\rightarrow$ aussi lentes que possible; uniformes $\rightarrow$ diffuses.

Entrées des évenements ensemble $\rightarrow$ un après l'autre. Prolonger le temps commun, respirer aussi lentement et profondément que possible, puis répartir le moins d'évenements possible sur un grand espace de temps, en laissant le plus de temps possible entre ces groupes.

Exemple 1b. 
2-Il est impossible, dans une telle partition, d'étudier les rapports qui se forment entre les différents paramètres, car ceux-ci vont se modifier d'une exécution à une autre.

3-Même si ces rapports demeuraient fixes, il se poserait quand même le problème du découpage des unités:

La notion de signe perd de sa précision et on ne peut repérer un signe minimal: il n'est pas possible, comme le voudrait Kowzan, d'établir une unité minimale de la représentation, qui serait comme une coupe dans le temps, échelonnant verticalement tous les codes; tel signe est ponctuel, éphémère (un geste, un regard, un mot), tel autre peut se prolonger tout au long de la représentation (tel élément du décor ou tel costume); le travail de démêlage des fils multiples des signes codés ne peut se faire qu'en utilisant des unités variables selon le code... (Ubersfeld 1978:32).

Il est à remarquer également qu'un seul paramètre peut s'organiser selon plusieurs codes simultanés. Le texte, par exemple, peut s'organiser à la fois selon le code langagier et selon le code musical; il en est de même pour la musique.

Dans les lignes qui précèdent, nous avons posé le problème de la correspondance entre les différentes étapes (partition(s) - exécution) d'une oeuvre ouverte. Mais qu'arrive-t-il si on décide de faire abstraction de ce problème et de considérer un de ces niveaux, la partition verbale par exemple, comme une entité en soi, et de limiter son étude à celle-ci? On s'aperçoit qu'à ce niveau, les différences et les isotopies ne sont pas pertinentes. Cela signifie qu'il n'y a pas de contexte préférentiel et que les différents éléments reviennent sensiblement le même nombre de fois pour une caractéristique donnée.

C'est donc dire que nous ne pourrons délimiter les particularités formelles de l'oeuvre que nous étudions, mais que nous devrons plutôt nous contenter de suggérer des modèles qui nous permettront de mieux en comprendre le fonctionnement.

\section{Trois modèles de « Glossolalie »}

Nous avons à cet effet tenté de trouver trois modèles qui pourraient rendre compte d'aspects différents du fonctionnement de Glossolalie. Le premier, celui du réseau, fait ressortir la mobilité des éléments qui composent cette oeuvre et montre comment chaque niveau de la partition forme un réseau qui ira s'imbriquer dans le réseau de niveau supérieur.

Le second modèle, celui de la grammaire générative, veut 
souligner la manière dont la conception de l'oeuvre rend possible, et surtout, exige la créativité de l'interprète.

Le dernier modèle, enfin, tente de mettre en évidence le caractère ludique de Glossolalie, l'ouverture suggérant déjà un certain jeu avec le concept d'oeuvre. Ce ludisme, la composition de Schnebel le concrétise par le jeu qui s'y instaure avec les mots ou la musique, ou encore entre les deux niveaux: musical et langagier.

\subsection{Glossolalie comme réseau}

Le modèle du réseau nous semble particulièrement bien adapté à la description de l'oeuvre que nous étudions puisqu'il représente une situation mobile qui change selon le chemin parcouru. De plus, dans le réseau, on peut délimiter des régions qui peuvent entretenir entre elles toutes sortes de rapports dont l'importance variera selon le cas.

Mais voyons tout d'abord la définition que nous donne Michel Serres du réseau:

Il est formé pour un instant donné (car nous verrons amplement qu'il représente un état quelconque d'une situation mobile) d'une pluralité de points (sommets) reliés entre eux par une pluralité de ramifications (chemins). Chaque point représente soit une thèse, soit un élément effectivement définissable d'un ensemble empirique (Serres 1968:11).

Dans Glossolalie, cette définition peut fonctionner à plusieurs niveaux, selon les points que l'on choisit. Premièrement l'ouvre entière peut être considérée comme le réseau des vingtneuf tableaux.

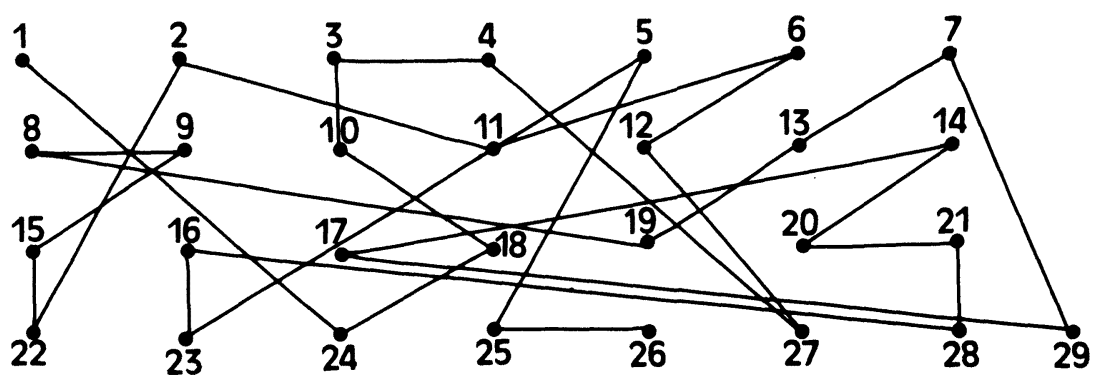


Dans ce modèle, chaque point a une importance égale et l'oeuvre exécutée sera plus ou moins considérable selon le nombre de tableaux que les interprètes vont réaliser, l'ordre pour ce faire n'ayant aucune importance.

On remarquera ici que Glossolalie se différencie à la fois des oeuvres où le hasard est total, comme c'est le cas pour une grande partie des oeuvres de John Cage par exemple, et des oeuvres où l'ouverture consiste en la permutation d'éléments fixes, comme dans le Klavierstück XI de Stockhausen. Dans Glossolalie en effet, il y a vingt-neuf entités dont chacune a son ouverture, mais qui sont malgré tout assez bien différenciées entre elles.

Si on passe à un niveau inférieur, les points représenteront les sections de chaque tableau.

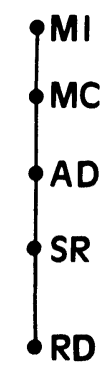

Exemple 3

Ce niveau constitue une exception, car l'ordre de lecture est obligatoire. On ne peut commencer par la dernière section pour ensuite passer à la première, chaque section venant ajouter des précisions à celle qui la précède.

C'est d'ailleurs à ce niveau que ressort une des caractéristiques essentielles de cette partition. Le hasard en musique est en général considéré comme un facteur instantané; nous voulons dire par là que dans la plus grande partie des oeuvres ouvertes en musique, le compositeur laisse à l'interprète une partition que celui-ci doit interpréter lors même de l'exécution sonore de la pièce, ce qui nous a valu le concept désormais courant de « happening ". Aussi bien chez Stockhausen que chez Cage, le caractère instantané de l'exécution est aussi important que la liberté de choix de l'interprète. On sait que c'est contre cette conception que s'est élevé Pierre Boulez dans son article "Aléa (1966). Pour ce dernier, l'acte de composer consiste justement à éliminer 
une quantité de solutions faciles qui se présenteront d'abord à l'esprit du créateur. Son rôle est donc avant tout de sélectionner parmi une grande quantité d'éventualités, rôle qui, selon Boulez, ne peut être assumé par l'interprète. Ce dernier, ne disposant d'aucun moment de réflexion, ne peut que choisir (sauf exception) la solution la plus facile ou la moins intéressante. On sait d'ailleurs combien souvent on a reproché aux " happenings " leur manque d'originalité et leur caractère de clichés. Dans la formation d'une telle oeuvre, l'interprète est, tout bien considéré, assez passif, puisqu'on ne lui demande que l'inspiration du moment:

Il se produit pourtant un phénomène négatif: les exécutants sont incapables d'inventer réellement quand on leur demande d'improviser dans un cadre plus ou moins limité. Même les personnes dotées d'un grand pouvoir d'invention ne font qu'utiliser leur répertoire d'habitudes instrumentales, de clichés personnels, de structures mémorisées (Globokar 1972:15).

Schnebel a semblé vouloir eviter cet inconvénient. Il a conçu une partition qui exige de la part de l'interprète un effort de construction de la pièce à exécuter, et demande même que de cette élaboration on fasse une seconde partition qui, elle, devra servir à l'exécution.

Ces considérations nous amènent à repenser les rôles de l'interprète et du hasard dans l'oeuvre que nous étudions. Globokar analyse comme suit la position de l'interprète dans la musique contemporaine:

En principe, en tant qu'interprète, j'ai toujours une tâche à remplir, plus ou moins complexe - créer le détail ou se référer aux conventions concernant "l'idée " ou la forme générale - et qui fut auparavant imaginée soit par le compositeur soit en commun accord par les interprètes présents. Cette tâche quelle qu'elle soit (conventions orales, ordre, suggestion, conseil, etc.), est constamment présente à ma pensée et c'est dans les moments de préoccupation et d'attention vers elle que je déverse automatiquement mes " habitudes » digitales ou stylistiques. Il en découle une question: est-il possible de décider d'être rationnel pendant un certain temps et de penser à la tâche à remplir, mais ensuite de ne plus y penser mais " décider " d'être instinctif et intuitif afin de pouvoir laisser libre cours à son imagination et inventer? Personnellement je pense que cela est actuellement impossible - du moins en Occident, et que c'est un vain espoir de croire que les musiciens pourront inventer quand on le leur 
ordonnera. Bien sûr ils produisent des sons et les combinent plus ou moins savamment, mais toujours dans le domaine du connu et en dehors des possibilités que l'on peut imaginer et projeter à une table de travail (ibid.:16).

Dans de tels cas, la difficulté nous semble provenir du fait que les compositeurs veulent partager la tâche avec des instrumentistes et non avec des musiciens. Un instrumentiste, de par la longue pratique de son instrument, a acquis un réservoir de réflexes qu'il lui sera difficile d'oublier, surtout s'il doit se fier à l'inspiration du moment. Et même si on le fait jouer sur un autre instrument que le sien, ce qui est souvent le cas dans la musique contemporaine, on lui demande simplement de "remplir» un espace de temps donné de manière presque automatique.

Schnebel au contraire considère l'interprète avant tout comme un musicien, et met à sa disposition un "manuel de composition », manuel à partir duquel devra s'opérer une série de décisions dans le sens où le décrit Jagodic-Davorin:

La décision, pour être opératoire, doit viser l'amélioration. C'est par cette croyance en une ligne commençant en un point donné à dépasser, pour aller atteindre un autre état de choses "meilleures", "plus vraies", que la décision est sous-jacente à tout projet métaphysique .... Dans chaque cas, elle va de pair avec une hiérarchisation qui est ainsi admise comme allant de soi; décider c'est analyser, séparer, imposer (Jagodic-Davorin 1972:55).

L'interprète qui aborde la partition de Glossolalie doit tout d'abord chercher à comprendre son mode de fonctionnement, analyser ensuite les différentes possibilités qui lui sont offertes, afin de prendre des décisions qui vont instaurer un certain ordre dans le matériel qu'il aura choisi. Dire ici que le compositeur partage la tâche compositionnelle, semble beaucoup moins métaphorique que dans les oeuvres où les interprètes ne font que se fier à leur inspiration du moment. En utilisant cette méthode, Schnebel a probablement voulu éviter une accumulation de clichés et de gestes traditionnels tout en laissant aux interprètes une liberté d'action considérable.

Ce genre d'écriture amène une collaboration particulière des interprètes entre eux. Ils doivent en effet concevoir séparément leur partie pour ensuite les coordonner entre elles. Ici encore, la partition demande un choix réfléchi; c'est donc véritablement une création collective (contrairement à l'improvisation de groupe où la pièce n'est formée que de la rencontre fortuite d'un ensemble d'événements). 
Mais revenons à notre réseau pour voir comment il s'applique au dernier niveau (voir ex. 4).

Nous ne donnons la représentation graphique que pour la première section $(\mathrm{MC})$ puisque le mode de fonctionnement sera le même pour chaque section. Ici, les sommets représentent les différentes caractéristiques énumérées par cette section. Si nous regroupions dans un même graphique toutes les caractéristiques énumérées dans les vingt-neuf tableaux, une section (la section MC par exemple) pourrait être comprise comme un chemin possible dans le réseau de l'ensemble des caractéristiques.

Chaque chemin est considéré comme un trajet parmi tous ceux qui sont possibles. Cela signifie qu'il n'y a pas de route nécessaire ou préférable à d'autres, ce chemin comprenant plus ou moins de caractéristiques selon le cas. Cette particularité explique pourquoi une analyse distributionnelle de cette pièce ne se révèle pas pertinente: à ce niveau de la partition, il n'y a pas de choix préférentiel ou exclusif.

D'autre part, nous pensons qu'il s'agit de la représentation formelle d'une situation mobile, c'est-à-dire qui varie globalement au cours du temps .... Dès lors, le chemin unique (ou l'ensemble des chemins sélectionnés) que choisissent la théorie, la décision, l'histoire - ou toute évolution donnée d'une situation mobile - est élu parmi d'autres possibles, déterminé parmi une distribution qui peut être aléatoire (Serres 1968:12).

Chaque point du réseau aura une portée plus ou moins considérable selon le chemin choisi (Schnebel définira des caractéristiques comme le degré de parenté des langues, la syntaxe qui sera dépravée ou courante, la sémantique qui sera cohérente ou insensée). La caractéristique qui définit la parenté des langues, par exemple, sera bien plus importante si elle est la seule à être définie. Si en plus de cela, la syntaxe, la sémantique et le domaine des langues sont également précisés, la première caractéristique perdra de sa puissance. De même, un réseau donné peut avoir une portée plus ou moins grande: lorsque le compositeur demande par exemple de faire ressortir la partie vocale ou instrumentale.

Serres décrit comme suit cette situation:

Dès lors, ce réseau est assez comparable à une sorte d'échiquier: sur ce dernier, il existe des pions à puissance équivalentes en droit, mais dont la puissance actuelle est variable selon leur situation réciproque à un moment donné, compte 


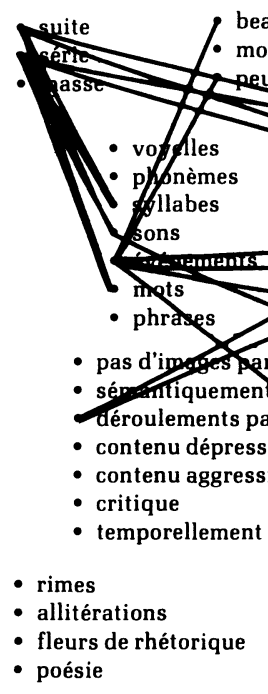

- grande

- moyenne

- petite

apparenté éloigné

langue maternelle

- langue quelconque

- durée

- dynamique

- hauteur
- haute sphère

- sphère quotidienne

- géographie

- littérature

- journaux

- impératifs

- menaces

- bavardages

- gémissements

- exclamations

- variable

- constant

peu variable

râclements de gorge

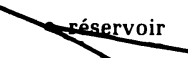

réservoir

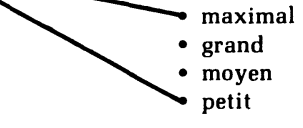

moyen

petit 
tenu de la disposition d'ensemble des pièces et de leur distribution complexe par rapport au réseau de jeu opposé, mais il existe aussi sur lui des pions à puissance différente (roi, dame, tour, cavalier...) qui sont sources (ou réceptions) de déterminations différenciées, par définition ou par nature, selon les chemins donnés (lignes, diagonales, colonnes, parcours brisés ...) mais dont la puissance dépend aussi (comme celle des pions équipuissants) de leur situation et distribution temporaire (ibid.:15).

Dans Glossolalie, les points, les sommets, auront des puissances différentes selon le niveau que l'on prend en considération: tableau, section ou caractéristique. Cette puissance variera également en fonction du nombre de points et de la position de chacun de ceux-ci. Etant donné que dans les premiers tableaux, certaines sections sont absentes, les autres en prendront donc d'autant plus d'importance.

En d'autres termes, il est possible de découper sur la totalité du réseau des sous-ensembles restreints, localement bien organisés, tels que leurs éléments soient plus naturellement référables à cette partie qu'à l'ensemble total (bien qu'en droit ils soient toujours référables à lui) (ibid.:16).

Cette particularité correspond au découpage par niveaux que nous avons déjà exposé. La totalité des caractéristiques forme un sous-ensemble des sections qui à leur tour forment un sous-ensemble des tableaux. On peut formuler autrement cette caractéristique en disant que de chaque point du réseau choisi à un niveau donné, on peut faire un autre réseau comme le démontre la figure de l'exemple 5 .

Une dernière particularité du réseau est l'interaction des sommets entre eux:

Mais la pluralité des connexions qui unissent les sommets impose à l'évidence l'idée d'une rétroaction, c'est-à-dire le retentissement immédiat de l'effet sur la cause, disons plutôt la rétroaction du sommet réception sur le sommet source (ibid.:20).

Ce phénomène se révèle particulièrement intéressant au niveau des vingt-neuf tableaux. Le premier tableau est une page vierge, ce qui veut dire en principe que l'interprète peut faire ce que bon lui semble. En réalité cependant, il est fort probable que cet interprète ait déjà lu les autres pages avant de réaliser le premier tableau. Il y a donc de grandes chances pour que cette lecture l'ait influencé dans la réalisation dudit tableau qui, par le 


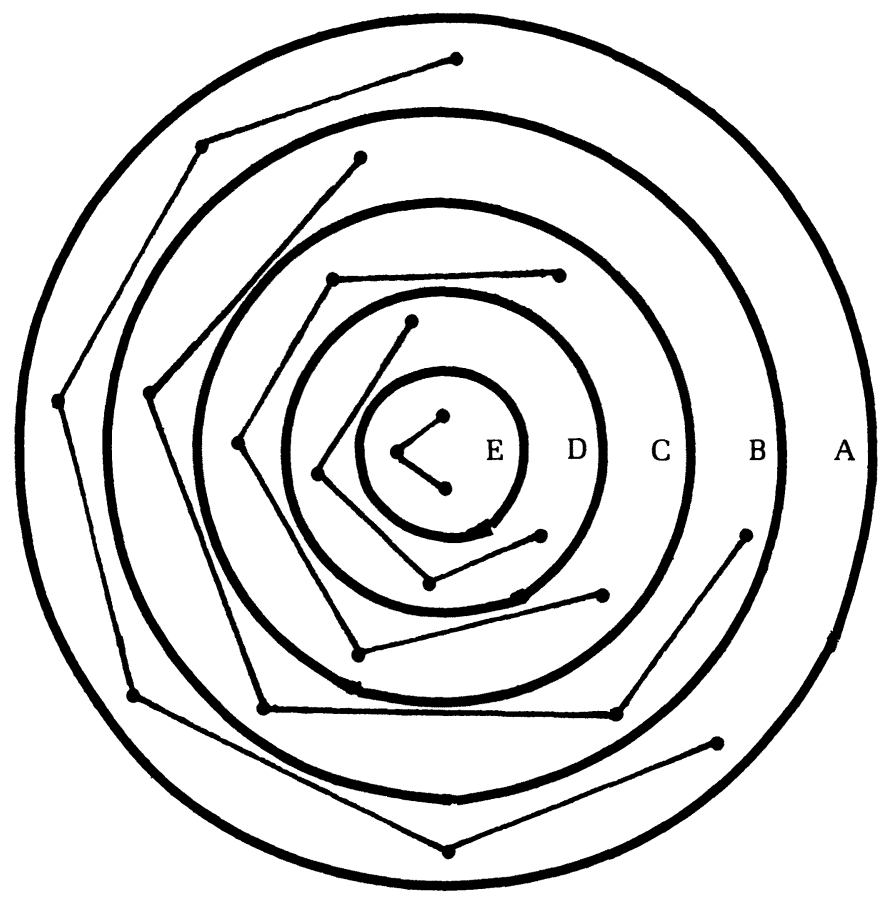

Exemple 5

( $A=$ tableaux,$B=$ sections, $C=$ caractéristiques, $D=$ valeurs des caractéristiques, $\mathrm{E}=$ interprétations de ces valeurs).

fait même, n'est plus aussi vierge qu'il n'y paraissait au premier abord. Il est également probable que la réalisation de chaque tableau élimine un certain nombre de choix pour les suivants, et de plus, il n'est nullement exclu que la réalisation par les interprètes du cinquième tableau par exemple, ne les oblige à apporter des modifications au premier ou au troisième.

Nous avons vu que le modèle du réseau, à cause de sa grande flexibilité, s'applique de manière fort pertinente à l'oeuvre que nous étudions et nous a permis de comprendre certains facteurs qui en constituent l'ouverture, comprise comme champ de possibilités multiples:

Tenir les deux bouts de la chaîne consiste à comprendre comment une transformation donnée va du probabilitaire au surdéterminé: au lieu de choisir arbitrairement une suite de déterminations fixes et équipuissantes, il faut ouvrir à gauche la détermination fixe en pluralités de sous- 
déterminations possibles, et à droite son univocité en surdétermination (ibid.:19).

\title{
3.2 « Glossolalie» comme grammaire générative
}

Passons maintenant à un autre modèle qui rend particulièrement bien compte d'un aspect différent de l'ouverture de Glossolalie: celui de la grammaire générative de Chomsky.

On sait que celle-ci a été élaborée en réaction aux thèses linguistiques qui la précédaient et qui ne rendaient pas compte d'une des particularités essentielles du locuteur: sa créativité. Dans l'optique de Chomsky, la langue est conçue comme un système pouvant engendrer une infinité d'énoncés:

\begin{abstract}
Alors qu'un corpus est par définition un ensemble fini d'énoncés, toute langue rend possible une infinité d'énoncés: puisqu'il n'y a pas de limite au nombre de propositions que l'on peut introduire dans une phrase française, on peut, à partir de tout énoncé français, en fabriquer un autre aussi régulièrement construit (en ajoutant, par exemple, une proposition relative): le distributionalisme est condamné par sa méthode à ignorer ce pouvoir d'infini inclu dans toute langue (Ducrot et Todorov 1972:57).
\end{abstract}

Chomsky voulait ainsi rendre compte, entre autres, du fait qu'un interlocuteur pouvait à la fois émettre des phrases dont la majorité est originale, et de même, reconnaître comme grammaticalement correctes des phrases qu'il n'avait jamais entendues auparavant. Il devait donc exister une certaine quantité de règles qui, tout en étant en nombre fini, permettraient de rendre compte de cette infinité d'énoncés.

On voit tout de suite l'intérêt d'un tel modèle pour l'oeuvre que nous étudions, oeuvre constituée d'un ensemble fini d'énoncés langagiers qui permettent une infinité d'énoncés musicaux.

Considérée de ce point de vue, la grammaire générative sera constituée par l'ensemble des règles contenues dans les vingtneuf tableaux, chaque tableau formant une dérivation particulière, effectuée par le compositeur lui-même ou par les interprètes, selon le cas, de cette grammaire.

On se souviendra qu'une grammaire générative, pour être acceptable, ne doit générer que les énoncés admissibles dans une langue donnée:

En quoi consiste, selon Chomsky, la description syntaxique (ou grammaire générative) d'une langue particulière? C'est un ensemble de règles, d'instructions, dont l'application 
mécanique produit les énoncés admissibles (=grammaticaux) de cette langue, et eux seuls (ibid.:58).

En conséquence, on pourrait nous objecter que notre application de ce modèle n'est pas valable, puisque la dérivation des règles contenues dans les vingt-neuf tableaux devrait produire une infinité de ces tableaux. On pourrait croire que la langue est finie, délimitée, car le corpus est justement constitué de ces vingt-neuf tableaux. La transposition n'est cependant pas aussi injustifiée qu'il n'y paraît tout d'abord, car le compositeur prend bien la peine de spécifier que le musicien n'est pas obligé de s'en tenir aux "préparations " (les vingt-neuf tableaux) fournies:

On peut également concevoir une composition de la pièce qui n'utilise aucune des "préparations " données et qui va au-delà du matériel fourni (Schnebel dans les explications qui précèdent la partition). ${ }^{5}$

Avant de passer à l'étude des règles proprement dites, il nous faut éclaircir un dernier point. La parition verbale, constituée des vingt-neuf tableaux, est effectivement un ensemble de dérivations effectuées par le compositeur. Rien ne dit cependant que la partition d'exécution et l'exécution elle-même, ne résulteront que des dérivations du compositeur, puisque, comme on vient de le voir, le fait que les interprètes puissent effectuer d'autres dérivations constitue la caractéristique qui a rendu possible l'application de ce modèle à Glossolalie.

Voyons maintenant comment, dans cette optique, on pourrait réécrire la partition verbale de Glossolalie.

Tout d'abord, l'oeuvre sera formée d'un nombre quelconque de tableaux:

(1) Glossolalie $\longrightarrow \# \mathrm{Ta}+\mathrm{Tb}+\ldots \mathrm{Tn} \sharp$ où $\mathrm{T}$ signifie tableau et $\mathrm{a}, \mathrm{b}, \mathrm{n}$, le numéro de ce tableau.

Chaque tableau se divise en sections:

(2) $\mathrm{T}$

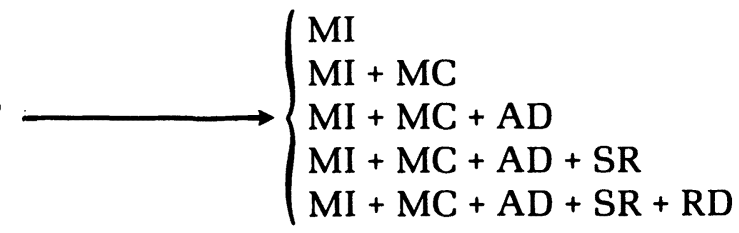

Ici, les lettres gardent la signification qu'elles avaient dans la description de la partition (i.e. $\mathrm{MC}=$ caractéristiques matérielles, $\mathrm{AD}=$ directives d'actions, etc....). 
Les sections suivantes se diviscat à leur tour:

(3) $\mathrm{MI}$ $\mathrm{H}+\mathrm{D}+\mathrm{Dy}$

( $\mathrm{H}=$ hauteur, $\mathrm{D}=$ durée, $\mathrm{Dy}=$ dynamique).

(4) $\mathrm{MC}$

(5) AD

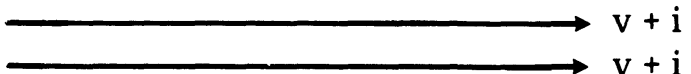

Avant de continuer, nous voudrions faire remarquer que toutes les règles (sauf la première bien entendu) sont facultatives, ainsi que les parties qui les constituent. On peut appliquer, ou pas, les règles.

On sait déjà que la section MI est graphique. En ce qui la concerne, on doit donc s'arrêter à ce stade.

Les sous-sections vocales et instrumentales ainsi que les sections non subdivisées se réécrivent en caractéristiques:

(6) $v(m)$ unité de déroulement + quantité d'articulations + proximité etc....

(La liste des caractéristiques est beaucoup trop longue pour que nous la donnions in extenso).

(7) $\mathrm{i}(\mathrm{m}) \longrightarrow$ unité de déroulement + etc....

(8) $\mathrm{v}(\mathrm{a}) \longrightarrow$ respiration + etc...

(9) $\mathrm{i}(\mathrm{a}) \longrightarrow$ temps + etc....

(10) SR $\longrightarrow$ succession des groupes + etc....

(11) RD $\longrightarrow$ espace de mouvement + etc....

Chaque caractéristique prend à son tour plusieurs valeurs:

(12) caractéristique $\rightarrow a+b+c+$ etc... les lettres représentant ici les différentes valeurs que peuvent prendre une caractéristique. L'unité d'articulation pouvant être par exemple un phonème (a), une syllabe (b), un mot (c) et ainsi de suite. On notera qu'à la différence des autres règles, une même caractéristique peut prendre plus de deux valeurs à la fois.

Nous donnons (ex. 6) la représentation graphique, sous forme d'arbre, d'une des dérivations qui résultent de ces règles, soit celle du tableau 15 de la partition verbale.

Qu'est-ce qu'un tel modèle nous apprend du fonctionnement de Glossolalie? Tout d'abord que la partition, comme une grammaire générative du langage, peut produire un nombre infini d'énoncés musicaux, ce qui nous renvoie au concept d'ouverture. Mais il faut remarquer que l'ouverture au niveau syntaxique n'est nullement créée par une ambiguïté quelconque, que 


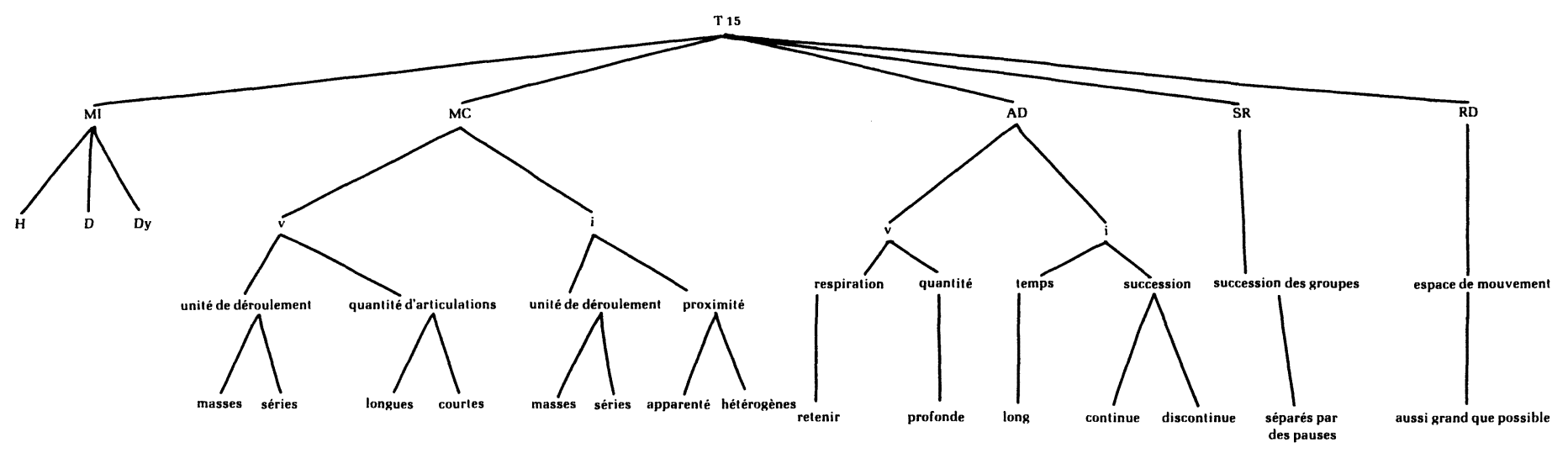

Exemple 6. 
ce soit celle des graphiques ou celle du langage. On peut faire l'analogie avec la grammaire du langage qui produit une infinité d'énoncés syntaxiques, la sémantique n'entrant nullement en ligne de compte à ce niveau. Il en est de même pour la partition verbale de Glossolalie qui, même si les symboles qu'elle utilise étaient univoques, n'avaient qu'une interprétation possible, pourrait produire une infinité d'énoncés syntaxiques.

Ce modèle permet également de comprendre que l'ouverture de cette oeuvre, au niveau syntaxique, dépasse la simple permutation des différents éléments qui composent la partition. Bien sûr, si on s'en tenait aux réarrangements rendus possibles par la partition, telle que laissée par le compositeur, le nombre en serait déjà presque illimité. Ce qui, à notre avis, est plus important cependant, c'est que le principe même de la partition de Schnebel permet un nombre infini d'ajouts, de prolongements et de raffinements, et ce, à tous les niveaux syntaxiques. Ce principe consiste à générer différentes catégories musicales, et il ne peut y avoir de limites à une telle production, puisque chaque individu a sa propre conception de la musique. De plus, toute évolution de la vie musicale produit de nouvelles catégories.

Le modèle de la grammaire générative fait également ressortir une autre caractéristique tout à fait particulière à cette partition, mais au niveau de son mode de production cette fois. On sait qu'aussi bien en linguistique qu'en musique, une grammaire générative est le produit de l'analyse d'un corpus donné, analyse le plus souvent contextuelle, de laquelle on déduit ensuite les règles qui gèrent l'enchaînement des différentes composantes. On peut de cette façon construire la grammaire générative d'un genre particulier ou encore celle de l'oeuvre d'un compositeur. Pour Glossolalie cependant, le processus est inversé: le compositeur semble donner le résultat de l'analyse d'un corpus fictif, analyse dont le musicien doit se servir pour produire un corpus réel cette fois. Ce phénomène suggère un jeu de renvois par une série de miroirs, où le rôle de chaque participant de la chaîne de production musicale est remis en question: le compositeur devient analyste, le musicien compositeur, et l'analyste se demande s'il ne doit pas d'abord composer avant de pouvoir analyser.

Si on peut concevoir Glossolalie comme analyse, on peut également se demander en suivant la même logique: analyse de quoi? Faut-il considérer la partition verbale comme l'oeuvre Glossolalie, la partition d'exécution ou l'exécution elle-même? 
Pour répondre à ces questions, examinons d'abord la pertinence de la distinction langue/parole de Saussure pour la musique:

Considérons...la production des sons nécessaires à la parole; les organes vocaux sont aussi extérieurs à la langue que les appareils électriques qui servent à transcrire l'alphabet morse sont étrangers à cet alphabet, c'est-à-dire l'exécution des images acoustiques n'affecte en rien le système lui-même. Sous ce rapport, on peut considérer la langue comme une symphonie dont la réalité est indépendante de la manière dont on l'exécute; les fautes que peuvent commettre les musiciens qui la jouent ne comprommettent en rien cette réalité (Saussure 1916:36).

La langue est ici comparée au système de l'oeuvre et la parole aux différentes exécutions, ce que Nattiez schématise pour une oeuvre traditionnelle de la manière suivante:

\begin{tabular}{|c|}
\hline langue \\
\hline parole \\
\hline
\end{tabular}

\begin{tabular}{|c|}
\hline œuvre \\
\hline interprétations \\
\hline
\end{tabular}

Exemple 7

Selon Nattiez, «cette comparaison du système de la langue à celui de l'oeuvre permet d'aborder le phénomène fondamental pour la compréhension du musical: celui de l'identité » (Nattiez 1975:77).

Selon la conception traditionnelle de la musique en effet, pour que l'exécution soit reconnue comme parole d'une langue (l'oeuvre), il faut que l'auditeur qui entend deux versions de la IIlième Symphonie de Beethoven puisse trouver entre les deux des traits communs: "Si nous pouvons reconnaître une même oeuvre à travers les diverses interprétations, c'est parce que, d'une version à une autre, quelque chose est conservé, c'est la pertinence des phénomènes sonores " (ibid.:79).

Ce qu'il est important de retenir ici, c'est que, chez Nattiez, pour que l'exécution soit reconnue comme équivalent de la parole saussurienne, il faut qu'un certain nombre de traits reviennent d'une exécution à une autre, traits qui seront alors considérés comme pertinents. Cette condition sera remplie si les transformations que chaque interprète fait subir à une oeuvre ne dépassent pas un certain seuil de tolérance de l'auditeur. 
Voyons maintenant ce qui se produit lorsqu'on applique ce modèle à Glossolalie. Si on veut retrouver dans la partition la somme des traits pertinents qui permettent à coup sûr de reconnaître l'exécution comme une réalisation de celle-ci, l'oeuvre devra alors se situer au niveau de la partition d'exécution:

\begin{tabular}{|l|}
\hline langue \\
\hline parole \\
\hline
\end{tabular}

\begin{tabular}{|c|}
\hline partition d'exécution \\
\hline exécutions \\
\hline
\end{tabular}

Exemple 8

Mais Nattiez nous dit que la dichotomie de Saussure peut, en musique, s'appliquer à un autre niveau: quand on considère le système de référence musical d'un style par exemple, comme la langue, l'oeuvre correspondra cette fois à la parole:

On peut cependant contester la schéma de Saussure et affirmer que s'il y a un système musical, il se situe au niveau des grands principes de la tonalité (ou de tel autre système de référence) dont les oeuvres ne sont que des "paroles" (ibid.:81).

Ce qui donne,le schéma suivant:

\begin{tabular}{|c|}
\hline langue \\
\hline parole \\
\hline
\end{tabular}

\begin{tabular}{|c|}
\hline système de référence \\
\hline oeuvre \\
\hline
\end{tabular}

Exemple 9

Cette fois, quand nous voulons appliquer le schéma à Glossolalie, on s'aperçoit qu'il manque une étape, puisqu'il semble difficile de comparer la partition verbale à un système de référence comme la tonalité. Nous devrons donc ajouter un niveau à ce schéma qui reste valable pour une oeuvre traditionnelle: 


\begin{tabular}{|c|c|}
\multicolumn{1}{c|}{ langue } & parole \\
\hline $\begin{array}{c}\text { système } \\
\text { de référence }\end{array}$ & oeuvre \\
\hline
\end{tabular}

\begin{tabular}{|c|c|c|}
\hline langue & parole & \\
\hline \multirow[t]{3}{*}{$\begin{array}{l}\text { partition } \\
\text { verbale }\end{array}$} & $\begin{array}{c}\text { partition } \\
\text { d'exécution }\end{array}$ & \\
\hline & langue & parole \\
\hline & $\begin{array}{l}\text { partition } \\
\text { d'exécution }\end{array}$ & exécution \\
\hline
\end{tabular}

Exemple 10.

On voit donc l'ambiguïté de la notion d'oeuvre. Si on s'en tient à la conception traditionnelle, on choisit de placer l'oeuvre au niveau de la partition d'exécution, partition dont on peut faire plusieurs réalisations sonores différentes. Mais dans ce cas, à quoi faire correspondre la partition verbale? Est-ce qu'un ethnomusicologue qui fait la transcription de plusieurs interprétations différentes (différentes par le rythme, la mélodie, etc...) de la même chanson dira qu'il a plusieurs chansons différentes? Qui doit décider où se situe le seuil qui, une fois franchi, interdit toute relation d'identité entre deux exécutions? De plus, l'absence d'identités, perceptibles ou analysables, ne permet pas automatiquement de refuser à la partition verbale le statut d'oeuvre. Il ne faut pas oublier en effet que, du point de vue du compositeur, Glossolalie correspond à une production unique, même si plusieurs réalités musicales totalement différentes sur le plan sonore, se réclament du même titre. Le compositeur, lui, n’a écrit qu'une Glossolalie.

\subsection{Glossolalie comme jeu}

Le dernier modèle que nous avons choisi pour illustrer le fonctionnement de Glossolalie est celui du jeu. Nous croyons intéressant de voir comment il fait ressortir à la fois l'évolution des rôles de l'interprète et du compositeur, et le principe de structuration de l'oeuvre qui nous occupe.

De nombreux philosophes et analystes ont appliqué ce con- 
cept à diverses manifestations artistiques, concept particulièrement pertinent pour décrire l'art contemporain:

Ces théories, qu'elles soient de provenance nietzschéenne ou husserlienne, heideggerienne ou linguistique, font du jeu un trait pertinent, terme auquel aboutit le penseur ou le créateur moderne qui regarde l'homme de la perspective de sa finitude. Le jeu sert bien ce qu'on pourrait appeler la perspective dissociative fondatrice de la conception sémiotique de l'homme telle qu'elle se laisse lire dans la sémiologie de Peirce et dans la linguistique de Saussure ou de Hjelmslev. Cette perspective dissociatrice renvoie à la rupture entre la présence humaine et une présence transcendantale, entre la musique des signifiants et la fiction des signifiés compris comme permanence d'un au-delà où se loge tranquillement le sens du monde (Krysinski 1975:43).

Afin d'expliquer les caractéristiques que nous venons de mentionner, nous avons choisi deux approches différentes du jeu, l'interprétation herméneutique qui nous suggère de nouvelles considérations sur les rôles respectifs du compositeur et des interprètes; puis une approche plus structurale qui nous apprendra quels éléments de l'oeuvre peuvent nous permettre une interprétation de celle-ci comme jeu.

Dans un premier temps, Gadamer nous dit que dans la conduite ludique, on ne peut séparer le joueur du jeu; ce joueur ne peut se comporter à l'égard du jeu comme s'il se trouvait face à un objet, sans que le jeu lui-même s'en trouve détruit:

Celui qui joue sait lui-même que le jeu n'est que jeu et qu'il se trouve dans un monde déterminé par le sérieux des buts. Il le sait, mais sans plus y penser, en tant que joueur, à ce rapport avec le sérieux. En effet, le jeu ne remplit son but que lorsque le joueur s'oublie dans le jeu (Gadamer 1976:27).

La finalité de tout jeu est celle de représenter. On joue toujours à quelque chose: les enfants vont se représenter une voiture, d'autres jeux vont faire allusion aux personnages de la cour. Cette caractéristique est également un des traits particulier de l'art: «Ce renvoi propre à toute représentation est comme mis en relief et devient constitutif de l'essence de l'art» (ibid.:35).

Pour cet auteur, c'est justement cet aspect représentatif qui abolit dans l'art la distinction entre acteur et spectateur, puisque: «L'ouverture au spectacle contribue à la clôture même 
du jeu: le spectateur ne fait qu'accomplir ce que le jeu est en tant que tel» (ibid.:35).

Il est intéressant de noter ici comment l'oeuvre ouverte pousse jusqu'à ses extrêmes conséquences cette caractéristique. Plusieurs de ces oeuvres, en effet, demandent une intervention active des spectateurs, que ce soit en leur demandant de manipuler les boutons d'un appareil stéréo ou en faisant évoluer différemment la pièce selon leurs interventions.

Une autre caractéristique fondamentale du jeu est son autonomie par rapport à ce qui rend possible son existence. Cette autonomie existe grâce à un phénomène que Gadamer appele la transmutation, terme par lequel il désigne la perte d'identité de ceux qui prennent part au jeu. Ce qui est important, ce n'est pas qui joue, mais bien ce qui doit advenir du jeu, toute l'attention se trouvant fixée sur la réalisation de ce jeu: «Ce qui n'existe plus, ce sont les joueurs - y compris l'écrivain et le compositeur » (ibid.:38).

L'oeuvre ouverte répond fort bien à cette description; dans la réalisation de Glossolalie, il est difficile de dire quelle part revient au compositeur et quelle part attribuer aux musiciens. Comme nous l'avons déjà vu, le compositeur partage avec les interprètes la composition de la pièce. Ce qui est important dans la réalisation de l'oeuvre n'est pas, comme c'est souvent le cas dans les oeuvres traditionnelles, quelle part revient au compositeur ou aux musiciens, mais la réalisation même de cette oeuvre. Ce partage de l'acte créatif semble d'ailleurs constituer un des traits importants de nombreuses créations contemporaines qu'on peut considérer comme ludiques. Il suffit de penser à l'usage fréquent de la citation par exemple dans de nombreuses oeuvres de Sanguinetti ou de Pound. On peut parler également des poèmes " renga " qui résultent de la collaboration de plusieurs auteurs (cf. Paz et al. 1971).

Une réalisation de l'oeuvre ouverte n'est qu'une des actualisations possibles de ce qu'on pourrait nommer l'analogon formel de toutes les réalisations contenues potentiellement dans la partition verbale. En parlant du jeu, Gadamer en vient à des conclusions semblables:

La "réalité" se tient toujours dans un horizon futur de possibilités désirées ou redoutées, en tout cas de possibilités encore ouvertes. Celles-ci sont telles que ne cessent de poindre ou de s'exclure mutuellement des attentes dont quelques-unes seulement peuvent être réalisées. C'est l'indé- 
cision du futur qui permet un foisonnement d'attentes tel que la réalité reste nécessairement en défaut par rapport à chacune d'elles (Gadamer 1976:39).

La principale caractéristique structurale qui va nous permettre de considérer une oeuvre comme jeu est sans doute celle de la fragmentation, chaque fragment représentant une entité plus ou moins autonome qui sera répétée dans des contextes variés au cours du déroulement de l'oeuvre:

Ce qui est ressenti comme jeu dans la poésie moderne renvoie souvent à la démarche structurante et génératrice à partir de quelques formules qu'on peut appeler séminales et dont le poète reconstruit à travers son texte la pertinence ou bien en propose une figuration (Krysinski 1975:50).

Parallèlement à cette première caractéristique, celle de la répétitivité joue également un rôle important dans les créations contemporaines à caractère ludique: "Le texte se fait ainsi comme combinatoire, comme jeu de citations dont la répétition marque le caractère ludique ou partiellement ludique du geste poétique " (ibid.).

On sait que ces deux traits sont deux caractéristiques importantes de l'oeuvre que nous étudions, où chaque élément est formé d'un ensemble de fragments. Par exemple chaque tableau est formé d'un ensemble de caractéristiques qui peuvent être considérées comme fragments puisqu'elles sont répétées telles quelles, ou légèrement modifiées, dans presque tous les tableaux. Ces tableaux forment à leur tour des fragments dont l'assemblage constituera la pièce. En principe, chaque fragment, qu'il s'agisse des caractéristiques ou des tableaux, peut être répété aussi souvent qu'on le désire.

On peut également souligner le caractère ludique de la relation qui s'établit, dans Glossolalie, entre musique et langage qui empruntent chacun au domaine de l'autre dans un va-etvient continuel entre les deux et où la frontière qui les départage est impossible à tracer.

\section{Oeuvre composée-oeuvre entendue}

Nous avons vu dans les pages qui précèdent que l'oeuvre ouverte occasionnait un changement considérable dans les relations entre compositeur, interprète et analyste. Mais elle produit également une modification appréciable des relations compositeur/public. 
On sait que le concept d'oeuvre ouverte a été appliqué à tous les arts (à ce sujet, voir Eco 1965). Une particularité de l'oeuvre ouverte en musique, cependant, la distingue de tous les autres arts: c'est la double réalité sous laquelle elle se présente à nous. Oeuvre composée d'un côté, oeuvre entendue de l'autre, le lien de vérifiabilité qui unissait la partition traditionnelle à son interprétation sonore n'existe plus. Nous nous retrouvons devant deux réalités souvent totalement différentes.

On se demandera donc dans un premier temps, quelle particularité de la musique rend possible l'existence de cette entité assez autonome qu'est la partition par rapport à son exécution. Une telle situation en effet n'est possible pour aucun autre art. Il est assez aisé de voir pourquoi il en est ainsi en peinture par exemple, en sculpture ou encore en architecture: il n'existe pas deux formes de représentation de la même oeuvre. Le théâtre fait ici exception puisqu'on a d'une part le texte et de l'autre la représentation.

Ce qui est particulier à la musique cependant, c'est qu'on peut rendre à volonté la correspondance entre les deux systèmes plus ou moins fixe. Nous voulons dire par là qu'un même signe graphique (par graphique nous entendons signe écrit, le signe peut être schématique ou langagier, il y a les deux dans Glossolalie) peut renvoyer à un nombre très restreint ou infini de réalisations sonores différentes, selon que le compositeur précise ou pas la manière dont on doit traduire ces signes. Cette situation est possible parce que les signes musicaux, contrairement aux signes langagiers, ne traduisent pas un système qui leur est extérieur: la sémantique. L'écrivain travaille avec un matériau qui possède deux niveaux bien distincts: le niveau sémantique, et celui des signes (écrits ou sonores); le sonore et l'écrit devant correspondre puisqu'ils renvoient tous deux au niveau sémantique. En littérature donc, un signe graphique ne peut renvoyer qu'à une réalisation sonore, ou pour être plus exact, à plusieurs réalisations qu'on considérera comme équivalentes au niveau de la signification:

On peut se demander comment l'écriture remplit sa fonction. Dans le domaine du langage, la fixation sur la signification du mot constitue le "tertium comparationis" entre le mot parlé et l'image écrite.... Il ne serait pas justifiable de considérer l'écriture comme simple résultat de la représentation, par des signes, des sons parlés (Georgiades 1977:79). ${ }^{6}$ 
En musique par contre, le compositeur n'a pas à tenir compte l'équivalences déjà fixées entre sonorité ou écriture et signification. Ceci permet l'existence d'un décalage beaucoup plus grand entre les structures sonores et écrites.

Dans Glossolalie, le compositeur a exploité cette particularité de l'organisation musicale, c'est en quoi cette composition se distingue de tous les autres genres d'oeuvres ouvertes, qu'il s'agisse d'oeuvres ouvertes par la simple permutation d'éléments par ailleurs fixés, ou encore d'oeuvres qui demandent la traduction en musique de signes graphiques pour lesquels on donne un code d'équivalence plus ou moins élaboré. Dans le premier cas, le compositeur peut créer un nouveau langage, mais il dit également à l'interprète comment l'utiliser, comment convertir en sonorités les signes de la partition. Dans le second cas, les signes graphiques peuvent facilement être traduits en langage musical conventionnel; rien en effet n'interdit d'interpréter une courbe sur une feuille comme une mélodie de Beethoven par exemple. En ce qui concerne Glossolalie, il est impossible de traduire la partition verbale par un langage musical traditionnel. Schnebel a précisément voulu rendre possible un nouveau langage, tout en en laissant l'articulation aux interprètes.

Cette constatation nous amène à l'affirmation de Ligeti qui dit en parlant de la musique sérielle et de l'oeuvre ouverte:

La ressemblance entre les deux méthodes se manifeste dès le début: elles opèrent toutes deux avec des directives. La méthode sérielle avec des directives de préformation d'opérations et d'ordonnements déterminés, la méthode aléatoire avec des directives pour des manipulations pré-ou para-musicales précises. Les dés de Cage, ses piles ou face, ses atlas célestes n'en sont pas moins des entreprises de préformation. Pour les prototypes de répartition des éléments, ce n'est pas la détermination ou l'indétermination de l'ordonnancement, ou de la répartition qui est déterminante pour le développement de ce qui a été pré-préparé, mais avant tout le fait qu'on ait considéré l'ordonnancement ou la répartition, quelle qu'elle soit, comme point de départ valable (Ligeti 1966:33). ${ }^{7}$

Pour Ligeti donc, le compositeur travaille tout d'abord avec un ensemble de directives qui le mèneront ensuite à la composition proprement dite; l'acte compositionnel est séparé en deux étapes bien distinctes (voir également Ligeti 1966:32).

Ces remarques nous semblent bien s'appliquer à l'oeuvre de 
Schnebel, le compositeur ayant élaboré les matériaux, laissant aux interprètes le soin d'élaborer les relations.

Encore ici, une telle procédure n'est possible qu'en musique. Il n'y a que dans cet art, en effet, où l'on puisse élaborer séparément matériaux et relations. Il est possible par exemple de concevoir un ensemble de relations auxquelles devront être soumises successivement plusieurs matériaux différents. Les compositions sérielles intégrales fonctionnaient un peu de cette manière: on déterminait une série que l'on appliquait ensuite à plusieurs paramètres musicaux (hauteurs, durées, timbres). Cette procédure est le contraire de celle qu'utilise Schnebel dans Glossolalie; il ne serait peut-être pas tout à fait injustifié de comparer cette oeuvre à une composition sérielle qui, au lieu de présenter une série de relations comme c'est le cas pour les compositions traditionnelles, présente une série de matériaux. En ce sens, on pourrait facilement imaginer une oeuvre ouverte où le compositeur se bornerait à inscrire sur la partition une ou plusieurs séries de relations abstraites que les interprètes devraient ensuite concrétiser par des matériaux musicaux. L'oeuvre serait ouverte puisqu'il n'y aurait aucun moyen de vérifier l'exactitude de l'utilisation de tel matériau plutôt qu'un autre.

La pratique musicale des dernières années $a$, comme nous l'avons vu, considérablement modifié la notion d'oeuvre. Ce changement place le musicologue devant un nouveau dilemme, car sa définition de l'analyse correspondait à la pratique traditionnelle qui mettait à sa disposition un corpus bien fixé et délimité. La définition de l'oeuvre musicale ayant évolué, le musicologue se doit lui aussi de resituer l'analyse à un niveau qui corresponde au nouveau corpus.

Les quelques considérations que nous avons exposées dans cet article se veulent une tentative dans cette direction qui, nous semble-t-il, peut encore mener à d'autres résultats intéressants en ce qui concerne la musique contemporaine. 


\section{NOTES}

1. La partition verbale de Glossolalie a été composée en 1959-60.

2. Schnebel a lui-même élaboré une partition d'exécution nomée Glossolalie 1961, publiée chez Schott's Söhne (No 6414). Il existe un enregistrement de cette version éditée chez Deutsche Grammophon (Avant-garde 3 DDG 643543). Glossolalie est prévue pour au moins quatre participants, récitants et instrumentistes, qui sont agissants, c'est-à-dire dont les déplacements sur scène sont eux aussi «composés».

3. L'exemple $1 \mathrm{~b}$ donne la traduction de l'exemple $1 \mathrm{a}$.

4. A ce sujet, cf. Seeger 1958.

5. «Denkbar wäre auch eine Komposition des Stücks, die keine der angegebenen Präparationen verwendet, sondern gerade die ausgesparten Materialbereiche durchführt ».

6. "Wie erfüllt aber Schrift ihre Funktion? Im Bereich der Sprache ist die Bindung an die Wortbedeutung das tertium comparationis zwischen gesprochenem Wort und geschriebenem Wortbild....Eine Schrift wäre nicht legitimiert, wenn wir sie lediglich als das Ergebnis einer Zeichengebung fur die Sprachlaute betrachten wollten ».

7. "Die Gemeinsamkeit bestand von Anfang darin, dass beide Methoden mit Direktiven arbeiten: die serielle Methode mit Direktiven der Vorformung determinierter Anordnungen und Operationen, die Zufalls-Methode mit Direktiven für bestimmte Manipulationen mit vor-musikalischen oder aussermusikalischen - Cages Würfel, Munzen oder Himmelsatlanten sind nicht weniger Vorformungsunterlagen als die bei Verteilungs-Mustern von Elementen ist nicht Determiniertheit oder Undeterminiertheit der Anordnung oder Verteilung für das weitere Schicksal des Vorbereiteten ausschlaggebend, sondern die Tatsache, dass überhaupt Anordnungen und Verteilungen - wie sie auch geschaffen seien - als verbindlich für die Ausgangsposition des kompositorischen Prozesses angenommen werden ». 


\section{RÉFÉRENCES}

BOULEZ, P.

1966: "Alea " (1958), in Relevés d'apprenti. Paris: Seuil, 41-56.

DUCROT, O. et TODOROV, T.

1972: Dictionnaire encyclopédique des sciences du langage. Paris: Seuil.

ECO, U.

1965: L'ouvre ouverte. Paris: Seuil.

GADAMER, H.G.

1976: Vérité et méthode. Paris: Seuil.

GEORGIADES, T.

1977: "Sprache, Musik, schriftliche Musikdarstellung, " in Kleine Schriften. Münchner Veröffentlichung zur Musikgeschichte, Bd 26, 73-80.

GLOBOKAR, V.

1972: «ils improvisent ... improvisez ... improvisons ...» Musique en jeu, $\mathrm{N}^{\prime \prime} 6,13-19$.

JAGODIC-DAVORIN, M.

1972: "Musique et décision, " Musique en jeu, $N^{\circ} 6,55-60$.

KRYSINSKI, W.

1975: «La dislocation des codes, le croisement des récits et la brisure de la représentation dans Six personnages en quête d'auteur de L. Pirandello ", Études littéraires, 495-514.

LIGETI, G.

1966: "Form, » Darmstädter Beiträge zur neuen Musik, X, 23-35.

NATTIEZ, J.-J.

1975: Fondements d'une sémiologie de la musique. Paris: Union générale d'editions.

PAZ, O., ROUBAUD, J., SANGUINETTI, E., et TOMLINSON, 1971: Renga. Paris: Gallimard.

SAUSSURE, F. de

1916: Cours de linguistique générale. Paris: Payot.

SEEGER, C.

1958: "Prescriptive and Descriptive Music Writing, " The Musical Quarterly, XLIV/2, 184-95.

SERRES, $M$.

1968: Hermès ou la communication. Paris: Les Éditions de minuit. UBERSFELD, A.

1978: Lire le théâtre. Paris: Éditions sociales. 